\title{
COVID-19 Lexicon in English News Reports Based on the Theory of Semantic Field
}

\author{
Mengxi $\mathbf{W u}^{*}$ \\ Southwest University, Chongqing 400715, China \\ *Corresponding author: Mengxi Wu, 1576022920@qq.com
}

\begin{abstract}
Coronavirus disease, or simply COVID-19, has affected many regions worldwide. The pandemic has caused great losses from all walks of life. Millions of people have died from the virus. In order to facilitate people's understanding of COVID-19, the present study adopts the theory of semantic field to analyze the COVID-19 lexicon that appeared in China Daily, an authoritative international daily newspaper issued by China. A total of 100 pieces of English news issued by China Daily have been randomly selected for this research. According to the theory of semantic field in structural linguistics, the meaning of a word cannot stand alone, but come into being with the meanings of its related words. Therefore, it is reasonable to try to understand COVID-19 as thoroughly as possible with relevant words, which form its semantic field.
\end{abstract}

Keywords: COVID-19; Lexicon; Semantic field; English news reports

Publication date: November 2021; Online publication: November 30, 2021

\section{Introduction}

Coronavirus disease, COVID-19 for short, has been hitting more and more regions worldwide since the end of 2019. It is necessary to read international news reports written in English. China Daily, an English newspaper published in China, has been chosen as the corpus of the present research for its high authority and popularity. As COVID-19 is an extremely serious situation concerning the whole world, the present research aims to provide people with a better understanding of COVID-19 from the perspective of lexicon under the guidance of the theory of semantic field in structural linguistics. News reports about the disease have been updating every hour. A total of 100 pieces of English news from China Daily have been selected for this research. As the analysis goes on, it has been found that the lexicon used in the news is relatively stable; thus, the sample size is suitable. This research may facilitate people's understanding of the pandemic from the perspective of humanities and social science.

\section{Literature review}

The foundation of the theory of semantic field, or simply called field theory, dates back to the mid-19th century in Germany, where two general linguists, Humboldt and Herder, laid the basis for field theory ${ }^{[1]}$. The concept of semantic field was first formally proposed and carefully studied by several structural linguists, such as Trier, in Germany and Switzerland in the 1930s. Ullman praised this theory as it opened up a new domain in the history of semantic research ${ }^{[2]}$. These pioneers have lain theoretical foundations for future studies on the semantic field, especially the application of field theory.

In the 20th century, linguists began to look into the depth of the semantic field of one particular word, rather than a large scope of many words. Even in the 21 st century, diachronic investigation has been conducted to review a certain Russian word in order to study its semantic field ${ }^{[3]}$. Similarly, the semantic field of the English word risk has been identified and analyzed ${ }^{[4]}$. Moreover, the study of semantic field 
could be combined with other theories, such as with semantic frames for historical exploration of the interface between language, action, and cognition ${ }^{[5]}$, with metonymy by corpus-based analysis ${ }^{[6]}$, or with communication strategies and policy practices ${ }^{[7]}$. The studies on semantic field have been successfully linked to other theories, so as to enrich the theories to study the human language. Liu applied field theory to a study to help Chinese students memorize English words ${ }^{[8]} . \mathrm{Li}^{[9]}, \mathrm{Guo}^{[10]}$, and $\mathrm{Li}^{[11]}$ acknowledged that field theory could work in college English vocabulary teaching. In the last few years, the study of semantic field varied according to the types of subjects, such as NBA lexicon ${ }^{[12]}$, Chinese outdated imperial examination lexicon ${ }^{[13]}$, and cooking lexicon ${ }^{[14]}$. Although these studies have been carried out from a new perspective, the application of field theory to vocabulary is the major aspect.

\section{Semantic fields of COVID-19 lexicon in China Daily}

Four semantic relationships, namely hyponymy, part-whole relationship, synonymy, and antonymy, have been adopted in the data analysis. Together with general semantic relationships, five specific semantic fields of COVID-19 in linguistic contexts within the 100 pieces of English news have been analyzed.

\subsection{Stages of the disease}

Five terms have been identified to describe the stages of the disease from the preparation period to its outbreak; they include run-up, imminence, incubation, diffusion, and rage. The five terms are hyponyms of their superordinate, period. The first stage, run-up, refers to the period before the virus finds a suitable host and attacks the living cells within the human body. The second stage, imminence, refers to the period when the virus enters the body and begins to replicate within the cells. The third stage, incubation, denotes the period when the virus manages to settle down in a host, but the host does not manifest any symptoms for a diagnosis to be made. As is reported, at this stage, there is a high chance that the infected host may infect others, and it is impossible to tell whether a person is affirmed or not. That is why people should be more alert and aware of this stage. The diffusion stage refers to the spread of the disease from one person to another. The fifth stage, rage, is a relatively colloquial word to describe the time when the virus has reached a transmissive peak in a massive population. Another semantic relationship can be found since news reporters use the informal word, rage, to describe the massive spread, while there is a relatively more formal term, outbreak, which combines with rage to form a synonymy. Besides, when reporters need to describe the transmission of the disease, they use epidemic, transmission, spread, dissemination, and diffusion, alternatively to denote the same conceptual meaning; thus, they are also in synonymy.

\subsection{Epidemic monitoring}

COVID-19 has many synonymous expressions in the news reports, which can be used interchangeably. They are coronavirus pandemic, coronavirus disease, COVID-19 pandemic, novel coronavirus, and novel coronavirus pneumonia, among which the last is a tentative term given by the National Health Commission of the People's Republic of China. The most formal and authoritative term used is COVID-19, given by the World Health Organization. In terms of real-time epidemic data, the common synonyms are data, figures, numbers, latest data, and fresh figures, which are used in the news when revealing different types of cases to describe the situation.

In the part-whole relationship of different cases to describe the epidemic situation, confirmed cases and suspected cases are the two types that concern the epidemic records the most. The virus has transmitted worldwide, and now, imported cases require careful watching. A more direct record of the disease can be acquired from the daily pandemic data. In sample news, people can view the colors of the map or read the updated tally in the pandemic situation to obtain information. 
On the other hand, a holistic and general description of the pandemic can be disclosed by terms denoting degree. For instance, terms about its risk level include high and medium; however, the term low cannot be found in the sample, implying that the virus is relatively more serious than the common virus, such as the influenza virus. In terms of specific symptoms, the modes of disease transmission require people's attention to prevent the spread of the virus. The modes can be divided into three major types: droplets, aerosol, and contact transmission. Each subcategory contains two to four types of detailed classification.

\subsection{Social reactions to the pandemic}

Most people are willing to come together to contain the pandemic, including officials, medical workers, and the ordinary people. Positive expressions of joint efforts include lockdown, report infections, consolidate strengths, confront, coordinate, and consult; they are in hyponymy. News reporters are actively revealing the truth of the pandemic situation in many aspects, such as report infections. The nation consolidates strengths to confront the virus by coordination and consultation. In terms of lockdown, faceto-face meetings have become less favored; instead, meetings are in the form of long-distance communication. For example, in hyponymous relations, news briefings proceed in the forms of televised news conference and online press conference. Moreover, meeting includes virtual meeting, and telephone conference is a type of virtual meeting. Certain social events can be seen from the terms pertaining to meetings described in the news; the outbreak of COVID-19 is one of them. Besides, there exist antonyms with different degrees in the description of tackling restrictions. News reporters collocate verbs such as impose, relax, or lift with the noun, restrictions, in which impose refers to laying down restrictions, lift means canceling restrictions, and relax refers to a middle situation between impose and lift. Therefore, impose and lift are in an antonymous relation.

\subsection{Medical reactions to the pandemic}

Medical lexicon related to the virus is complicated and may be unfamiliar to common people, so the understanding of this semantic field needs due attention from news readers. For example, a mask is a necessity for an individual to go out. The types of expressions of mask found in the sample are in hyponymy, with the word face mask being the superordinate of the other two words. Another group of hyponyms and hypernyms is more complicated for its contribution to overcoming the pandemic. The word medical force contains many hyponyms. The medical force mainly includes staffs and materials, or team and equipment, alternatively. News reports contain many terms related to the medical force because the infected needs cure and treatment, not death. People all around the world have to spend a substantial amount of money to protect themselves and save other people's lives. Besides, reservoir refers to a population or tissue which is chronically infested with the causative agent of a disease and can act as a source of further infection. They are in a hyponymous relation, with infected organisms being the superordinate. In the sample news, the expressions of medics, medical workers, medical staff, and medical team can be used interchangeably because they are synonyms in the COVID-19 contexts.

\subsection{International mutual assistance}

International assistance is coming from all around the world in showing sympathy for those people who are suffering. Therefore, international mutual assistance has become a precious and important measure to contain the pandemic. For example, in the sample, Sittard-Geleen is termed sister city by Guangdong Province, South China. The pair of synonyms discloses that international mutual assistance is based on kindness for all human beings. In a hyponymous relation, international assistance sent from China is the 
superordinate of other words. These experts and materials are sent by chartered planes and in combination with private jets, they form hyponyms of the word plane. Besides, food is of vital importance in this pandemic. The news reports revealed chaos with the crazy purchase of daily necessities in some supermarkets. That is why food assistance is needed by other coronavirus-hit regions. In an antonymous relation, dispatch and introduce reveal two aspects of the semantic field of international mutual assistance. One party sends out expert teams or materials to help badly-hit regions in tackling the disease, while another party introduces staffs and supplies in its epicenter. This proves the relatively harmonious atmosphere in such a cruel pandemic situation by cooperation among the countries around the world.

\section{Conclusion}

In the present study, the author adopts the theory of semantic field to analyze the lexicon related to COVID19 in English news reports, so as to facilitate people's understanding of the pandemic from the perspective of lexicon. The analysis has been made within the framework of four semantic relationships and five semantic fields categorized by the author's interpretation of the contexts in the news. The semantic fields include the stages of the disease, epidemic monitoring, social reactions to the pandemic, medical reactions to the pandemic, and international mutual assistance. The present study may present certain educational value, providing people with better understanding of the pandemic by linking the lexical meaning of words together.

\section{Disclosure statement}

The author declares that there is no conflict of interest.

\section{References}

[1] Wen X, 1995, A Study of the Fuzziness of Language by Field-Theory. Foreign Language Research, (01): 27-32, 80 .

[2] Ullmann S, 1962, Semantics: An Introduction to the Science of Meaning, Basil Blackwell, Oxford, Britain.

[3] Vesnina G, 2014, Lad and Ladit' in Lexical-Semantic Field 'Norm'. Procedia - Social and Behavioral Sciences, 15428: 233-236.

[4] Boholm M, 2017, The Semantic Field of Risk. Safety Science, 92: 205-216.

[5] Nerlich B, Clarke D, 2000, Semantic Fields and Frames: Historical Explorations of the Interface between Language, Action, and Cognition. Journal of Pragmatics, 32(2): 125-150.

[6] Ädel A, 2014, Metonymy in the Semantic Field of Verbal Communication: A Corpus-Based Analysis Of Word. Journal Of Pragmatics, 67: 72-88.

[7] Stevens T, Aarts N, Dewulf A, 2019, The Emergence and Evolution of Master Terms in the Public Debate About Livestock Farming: Semantic Fields, Communication Strategies and Policy Practices. Discourse, Context \& Media, 31: 100317.

[8] Liu L, 2004, Sementic Field Theory and English Vocabulary Study. Journal of Tangshan College, (03): 73-74, 90.

[9] Li Y, 2006, The Enlightenment of Semantic Field Theory to College English Vocabulary Teaching. Journal of Yibin University, (10): 85-87.

[10] Guo C, 2010, The Application of the Semantic Field Theory in College English Vocabulary Instruction. Chinese Journal of Applied Linguistics, 33(03): 50-62, 31, 126. 
[11] Li Y, 2012, The Application of Semantic Field Theory in College English Vocabulary Teaching. Journal of Shenyang Normal University (Social Science Edition), 36(05): 142-144.

[12] Wang Y, 2017, Lexical Semantic Field in NBA Language. Overseas English, (13): 3-4.

[13] Zhang L, 2017, A Study of Imperial Examination Vocabulary in Ming and Qing Dynasties Based on Semantic Field Theory. Jianghuai Tribune, (05): 170-176.

[14] Wang L, 2019, A Contrastive Study of the Semantic Fields of English and Chinese Cooking Words. Overseas English, (04): 97-98. 